\title{
Experimental research of the mechanism and particle flow in screw conveyer
}

\author{
Meiqiu Li, Jingbo Luo, Bangxiong Wu, Jian Hua* \\ School of Mechanical Engineering, Yangtze University, Jingzhou 434023, China
}

Corresponding Author Email: huajian5410@yangtzeu.edu.cn

https://doi.org/10.18280/ijht.360123

Received: 21 September 2017

Accepted: 2 November 2017

\section{Keywords:}

screw conveyor, outlet mass flow rate, fill rate, trajectory angle, particle flow.

\begin{abstract}
This paper aims to disclose the mechanism of the screw conveyor and detail the particle flow inside this type of conveyor. To this end, the author discussed the relationship between the trajectory angle of particle motion and several key parameters based on the fluid mechanics. Then, a theoretical model of particle flow was established for screw conveyor of sand, considering the lateral sliding efficiency, the fill rate and the theoretical maximum mass flow rate. The theoretical analysis shows that the outlet mass flow rate depends on such parameters as the inclination angle, rotation velocity and pitch. Through several tests, the author obtained the variation patterns of the fill rate with rotation velocities and pitches, and those of the outlet mass flow rate with inclination angles, rotation velocities and pitches. After that, the change law obtained by our theoretical model was contrasted with that observed in Reference. The comparison shows that the two laws are basically the same. Thus, the proposed model is accurate enough to be applied in the practical design of the screw conveyor.
\end{abstract}

\section{INTRODUCTION}

Since the boom in oil production in the 1950s, the oil industry has evolved into a pillar of China's national economy. Nowadays, however, some major oilfields in China have entered the stage of later exploitation, and witnessed a decrease in the oil production efficiency. To improve the oil recovery rate, one of the common methods is to perform the fracturing operation [1]. The success of the operation relies heavily on the fracking fluid, a mixture of water, sand and chemicals.

Before the fracturing operation, the sand of the fracking fluid needs to be transported on a screw conveyor. Considering the limited space of oil wells, it is necessary to enhance the conveying efficiency of the screw conveyor as much as possible. To do so, the focal and difficult points include determining the mix ratios of liquid and sand, and deriving a theoretical formula for the sand mass flow rate of the screw conveyor [2]. If conveyed at a high fill rate, the discrete mixture can be regarded as the ideal fluid to simplify the analysis of its flow law during the transport. The fill rate refers to the amount of particles that can be conveyed in the screw conveyer. Nevertheless, there is still an essential difference between the actual mixture and the ideal fluid.

So far, most scholars have been concentrating on horizontal and vertical spiral conveyors. In general, the theories on the mechanism of the spiral conveyor fall into three categories: single point theory, fluid mechanics theory and particle swarm theory. Taking the single point theory for example, Emhremidis, Sinha and Vierling [3] were the first to apply the single point theory to study the mechanism. Specifically, the material attached to the inner wall surface was simplified as a single particle for dynamic and kinematic analysis. The analysis shows that the material is thrown onto the inner wall by the centrifugal force generated from the fast-rotating spiral shaft, and is affected by the surface friction of the spiral blade; the material is dynamically balanced by gravity in the vertical direction, the surface friction of the cylinder wall, the surface friction of the blade, the support force on the blade, the centrifugal force and the support force on the cylinder wall. In light of these, these scholars established a dynamic equilibrium equation for the material, obtained the critical velocity equation of the particle, and determined the outlet mass flow rate and power consumption formulas of the conveyor.

By contrast, there are only a few reports on the screw conveyor. Song [4] explored the conveying efficiency of horizontal screw conveyor and material movement mechanism during the conveyance, pointing out that the fill rate is a key parameter of the screw conveyor. Huang [5] investigated the conveying mechanism of screw conveyor in the paper industry, and determined the conveyor velocity, screw shaft diameter, screw blade diameter and screw pitch for paper-making. Hu [6] proposed the theoretical formula of outlet mass flow rate, conveyor parameters and power consumption of the screw conveyor, and presented a design for wear resistance during blade manufacturing. $\mathrm{Xu}$ [7-9] probed deep into the screw conveyor in brick, rubber and cement industries, and identified the factors influencing the conveying efficiency of the conveyor. Following fluid mechanics theory, Nilsson and Rademacher [10] examined the mechanism of the screw conveyor, especially that with a high fill rate. Taking the particle flow in the conveyor as an ideal fluid, these scholars set up the curved surface equation, the moment balance equation and the pressure distribution equation of the fluid according to its law of movement.

Thus, it is unsurprising that the traditional formulas and test results fail to suit the screw conveyor. If these formulas or results are followed blindly in the conveyor design, there will be dead-zones in the flow of the materials, leading to clotting and even machine failure. For instance, the abovementioned single particle theory, which ignores the 
interaction between particles, only applies to the screw conveyor with a low fill rate. If it is applied to a high-fill rate screw conveyor, the theoretical results will deviate far from the actual conveyance. This calls for feasible solutions that can elevate the conveying efficiency of the screw conveyor, and a theoretical calculation method for the material flow rate on the screw conveyor [11]. For the screw conveyor, the conveying efficiency depends on its diameter, screw pitch, inclination angle, friction coefficient, blade-wall clearance, etc. [12]. The interactions among these parameters directly bear on the conveying mechanism of the screw conveyor.

\section{VELOCITY ANALYSIS}

During the normal operation of the screw conveyor, the particle distribution and type of free surface are the same with those of the spiral conveyor. The curve of the free surface is parallel to the curve of the spiral blade. Figure 1 illustrates the velocities and forces of particles of a stable sand conveyor, in which the trajectory of the particles is opposite to the rotation direction of the spiral blade. In Figure $1, V_{B}$ is the circumferential velocity of the spiral blade, $V_{R}$ is the velocity of particles relative to the blade surface, $V_{A}$ is the absolute velocity of the particles, $\alpha$ is the spiral angle of the blade. The angle $\lambda$ is the same as the absolute velocity direction, which indicates the spiral angle of particle motion.

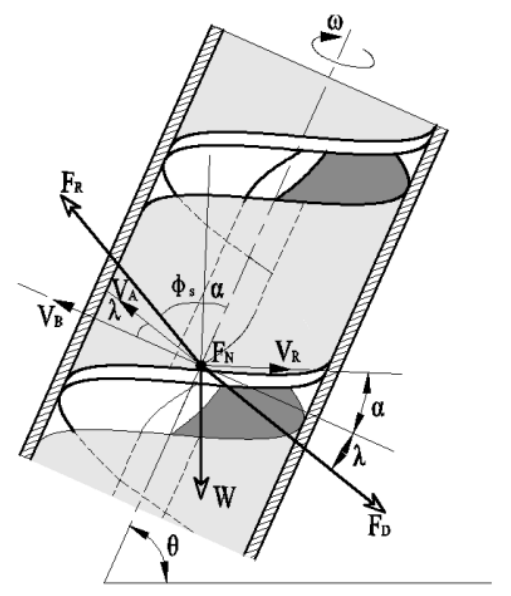

Figure 1. Velocity and force analysis

The blade velocity at a distance $r$ from the centre of the screw shaft can be expressed as:

$$
\begin{aligned}
& V_{B}=r \omega \\
& \omega=\frac{\pi n}{30}
\end{aligned}
$$

where $\omega$ is the angular velocity of the screw shaft; $n$ is the rotation velocity of the screw shaft $(\mathrm{r} / \mathrm{min})$.

According to the velocity relationship, the velocity of particles relative to the blade surface can be obtained as:

$V_{R}=\frac{V_{B} \sin \lambda}{\sin (\alpha+\lambda)}$

The relative velocity relies on the friction force from the inner wall of the spiral cylinder, which itself hinges on the friction coefficient between the wall and the particles and the centrifugal force generated from the rotating spiral shaft. Hence, the vector relationship of the absolute velocity of the particles can be expressed as:

$\vec{V}_{A}=\vec{V}_{B}+\vec{V}_{R}$

The absolute velocity of the particles can be obtained by Equations (2) and (3):

$V_{A}=\frac{V_{B} \sin \alpha}{\sin (\alpha+\lambda)}$

According to the velocity relationship, the absolute velocity can be decomposed into the effective delivery velocity $V_{Z}$ along the axis of the spiral shaft and the rotation velocity $V_{F}$ along the tangent line:

$V_{Z}=V_{A} \sin \lambda$

$V_{F}=V_{A} \cos \lambda$

The effective delivery velocity of the particles can be obtained by Equations (4) and (5):

$V_{Z}=\frac{V_{B} \sin \alpha \sin \lambda}{\sin (\alpha+\lambda)}$

The circumferential velocity of the particles along the tangent line can also be obtained by Equations (4) and (5):

$V_{F}=\frac{V_{B} \sin \alpha \cos \lambda}{\sin (\alpha+\lambda)}$

The relationship between the velocities of the particles is shown in Figure 2.

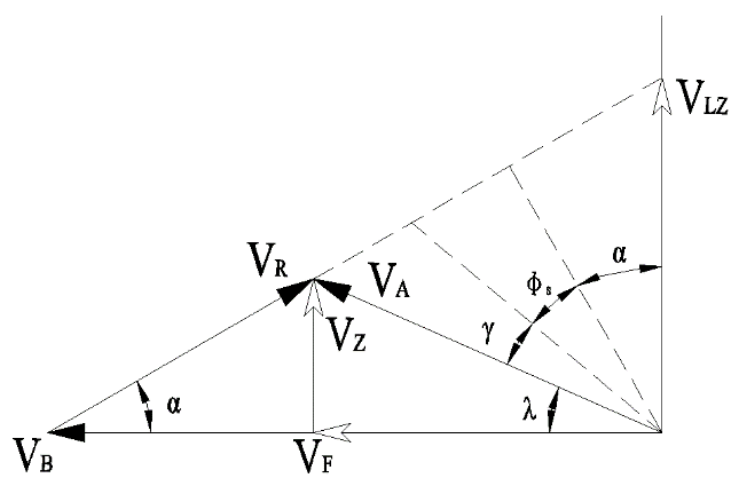

Figure 2. The relationship between the velocities of particles

\section{OUTLET MASS FLOW RATE}

\subsection{Pressure distribution}

The lateral pressure of the pile of granular particles (hereinafter referred to as the granular pile) obeys the distribution specified in Reference [13]. Let $h$ be the distance of the granular pile from the free surface. Then, the vertical pressure of the granular pile at the distance of $h$ can be expressed as: 
$p_{v}=\rho g h$

where $\rho$ is density of particles; $h$ is the height of the granular pile from the free surface.

The lateral positive pressure of the granular pile is

$p_{r}=k_{j} p_{v}$

where $k_{j}(0.4 \sim 1.0)$ is the lateral pressure coefficient of the granular pile.

Our research object is a circular ring unit of the granular pile. The lateral positive pressure of the ring at low rotation velocities can be expressed as:

$F_{a}=k_{s} W$

During the conveyance of sand, the actual trajectory of the ring is equivalent to an ellipse (Figure 3). The particles are moving at an absolute velocity $V_{A}$ over a curved path with a radius of curvature equal to the maximum radius of the ellipse $R_{c}$ :

$R_{c}=\frac{r}{\cos ^{2} \lambda}$

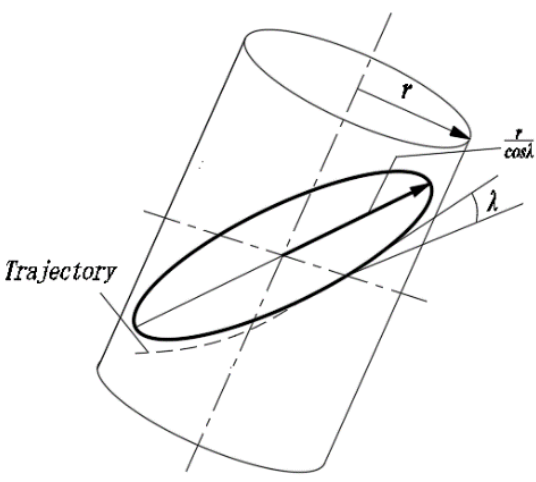

Figure 3. The trajectories of particles

Let us denote the mass of the sand in a ring segment as $\mathrm{m}$. Then, the centrifugal force of the ring segment during the circular motion $F_{c}$ can be expressed as:

$F_{c}=\frac{m v_{A}^{2}}{R_{c}}$

Substituting Equation (1) into Equation (12), we have:

$F_{c}=\frac{W \omega^{2} r}{g}\left(\frac{\tan \alpha}{\tan \alpha+\tan \lambda}\right)^{2}$

During the steady flow of the particles, the pressure of the granular pile against the cylinder wall is composed of the centrifugal force and the static lateral pressure:

$F_{N}=F_{c}+F_{a}$

Substituting Equations (10) and (13) into Equation (14), we have:
$F_{N}=W\left[k_{s}+\frac{W \omega^{2} r}{g}\left(\frac{\tan \alpha}{\tan \alpha+\tan \lambda}\right)^{2}\right]$

where $F_{D}$ is the boundary friction force, which pointing to the opposite direction of absolute velocity.

$F_{D}=\mu_{g} F_{N}$

Substituting Equation (15) into Equation (16), we have:

$F_{D}=\mu_{g} W\left[k_{s}+\frac{\omega^{2} r}{g}\left(\frac{\tan \alpha}{\tan \alpha+\tan \lambda}\right)^{2}\right]$

\subsection{Trajectory angle of particle motion}

As shown in Figure 1, the force balance of the particles can be obtained as:

$\left\{\begin{array}{l}W \sin \theta+F_{D} \sin \lambda=F_{R} \cos \left(\alpha+\phi_{s}\right) \\ F_{R} \sin \left(\alpha+\phi_{s}\right)=F_{D} \cos \lambda+W \cos \theta\end{array}\right.$

Substituting Equation (17) into Equation (18), we have:

$\mu_{g}\left[k_{s}+\frac{\omega^{2} r}{g}\left(\frac{\tan \alpha}{\tan \alpha+\tan \lambda}\right)^{2}\right]$

$=\frac{\cos \theta-\sin \theta \tan \left(\alpha+\phi_{s}\right)}{\sin \lambda \tan \left(\alpha+\phi_{s}\right)-\cos \lambda}$

According to Equation (19), the trajectory angle of the granular pile is affected by such factors as rotation velocity, radius, pitch, inclination angle, boundary friction etc. Assuming that the boundary materials of the screw conveyor remain constant, the rotation velocity, pitch and inclination angle were taken as the controllable parameters of the screw conveyor. Here, the inclination angle is set to $45^{\circ}$, the rotation velocity to $400 \mathrm{r} / \mathrm{min}$, and the pitch to $50 \mathrm{~mm}$. Then, $k_{s}$ was determined as 0.9 according to the corollary in Reference [13]. It can be seen from Figure 4 that the particle trajectory angle of the circular ring unit increases with the radius at a gradually decreasing rate.

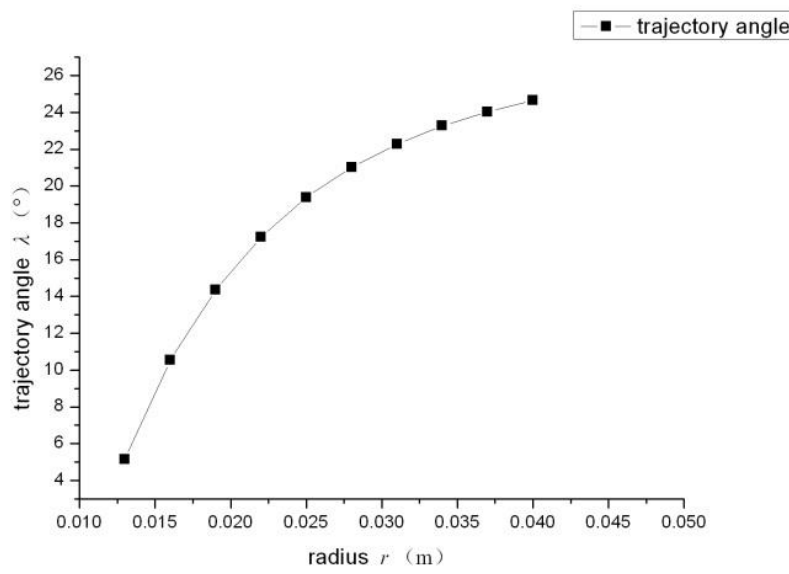

Figure 4. Variation of the trajectory angle with radiuses

Below is an investigation into the effect of rotation velocity, pitch and inclination angle on the trajectory angle of 
the granular pile. First, the trajectory angles of particles were calculated for different radiuses; then, the particle stacking angles at five positions on the radius of the spiral blade were averaged as the particle trajectory angle. Let $R_{0}$ be the radius of the spiral shaft, $R$ be the radius of the cylinder wall, $r_{i}$ be the distance of the circular ring unit from the spiral shaft, and $\lambda_{i}$ be the trajectory angle of the unit at the distance of $r_{i}$. Then, five distances were respectively determined as:

$$
\begin{aligned}
& r_{1}=R_{0} \quad r_{2}=\frac{3 R_{0}+R}{4} \quad r_{3}=\frac{R_{0}+R}{2} \\
& r_{4}=\frac{R_{0}+3 R}{4} \quad r_{5}=R
\end{aligned}
$$

The values $r_{i}$ were substituted into Equation (19) to get the trajectory angles $\lambda_{i}$ at the five positions. Then, the final trajectory angle of the particles can be obtained as:

$$
\lambda_{a v}=\sum_{i=1}^{5} \lambda_{i}
$$

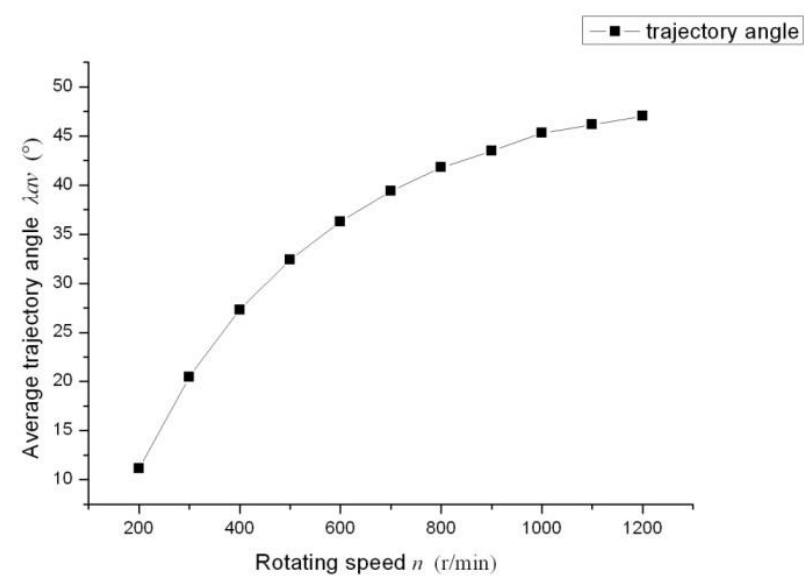

Figure 5. Variation of the trajectory angle with rotation velocities

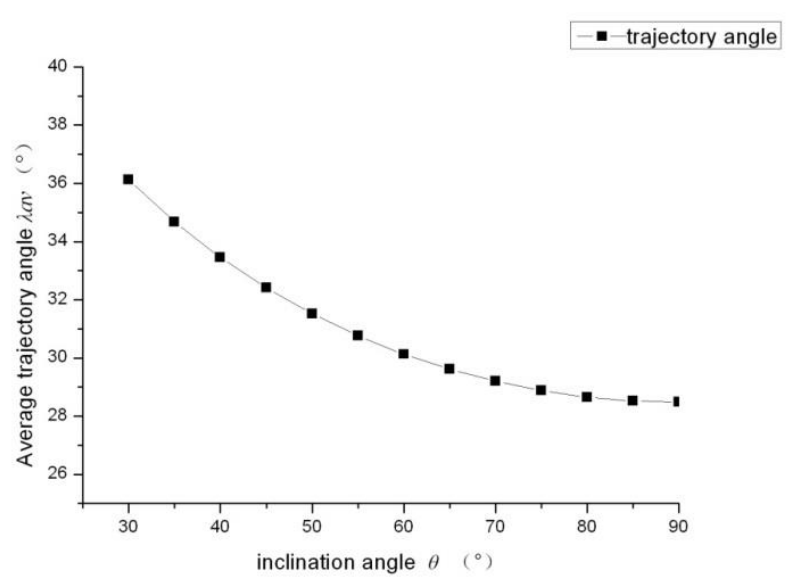

Figure 6. Variation of the trajectory angle with inclination angles

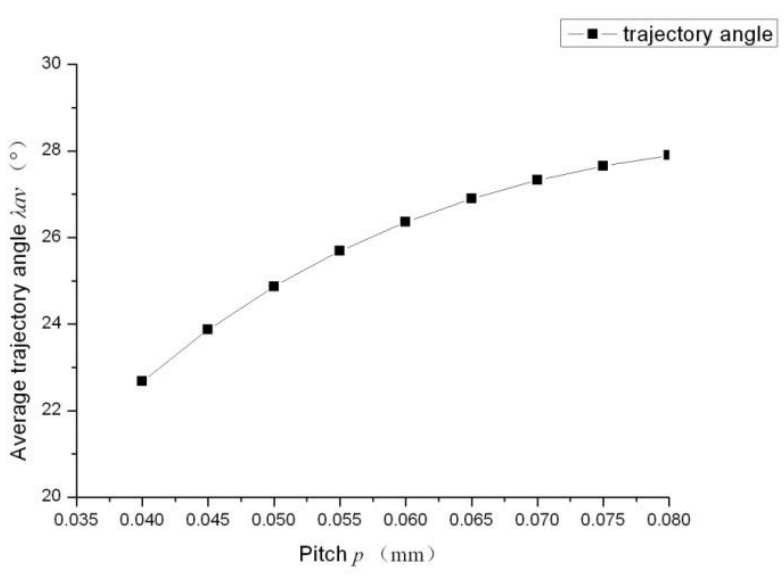

Figure 7. Variation of the trajectory angle with pitches

Similarly, it is discovered that the trajectory angle increased with rotation velocity at a decreasing rate at the inclination angle of $45^{\circ}$ and the pitch of $50 \mathrm{~mm}$ (Figure 5). It is also observed that the trajectory angle decreased with the increase of the inclination angle at the rotation velocity of $400 \mathrm{r} / \mathrm{min}$ and the pitch of $50 \mathrm{~mm}$; however, the decreasing rate approached 0 at the inclination angle of $70^{\circ}$, and reached 0 when that angle fell between $70^{\circ}$ and $90^{\circ}$ (Figure 6). Moreover, the trajectory angle increased with the pitch at a decreasing rate at the rotation velocity of $400 \mathrm{r} / \mathrm{min}$ and an inclination angle of $45^{\circ}$ (Figure 7).

\subsection{Relative slippage efficiency}

During the steady conveyance of the particles, there is a relative slippage between the particles and the blade surface under the shear action. Because of the resulting upward motion of the particles, the circumferential velocity of the particles falls below that of the blade edge. The efficiency of the relative slippage, i.e. the amount of slippage of the particles relative to the blade, can be understood as the ratio of the actual conveying velocity of the particles to the maximum theoretical conveying velocity:

$\chi=\frac{V_{Z}}{V_{L Z}}$

According to the velocity relationship, we have:

$\chi=\frac{\tan \lambda}{\tan \lambda+\tan \alpha}$

\subsection{Mass flow rate}

The quality of the fracking fluid directly hinges on the efficiency and accuracy of sand conveyance. Thus, the volumetric flow rate of the screw conveyor is the key parameter to both the fracturing operation and the conveyance process. This parameter can be expressed as:

$Q=Q_{T} \psi$

where $Q_{T}$ is the theoretical maximum mass flow rate of the screw conveyor, that is, the mass delivered by all the particles in a single pitch (provided that all the particles are 
filled in a single pitch and the screw shaft rotates one revolution); $\Psi$ is the volumetric efficiency of the granular pile during conveyance. The size parameters of the screw conveyor are shown in Figure 8, where $D_{i}$ is the diameter of the blade edge, $D_{o}$ is the diameter of the spiral shaft, $p$ is the pitch, $t$ is the blade thickness, $c$ is the blade-wall clearance, and $\theta$ is the inclination angle. Therefore, the theoretical maximum mass flow rate of the screw conveyor can be expressed as:

$$
Q_{T}=\frac{\pi}{4} n\left[\left(D_{i}+2 c\right)^{2}-D_{o}^{2}\right](p-t)
$$

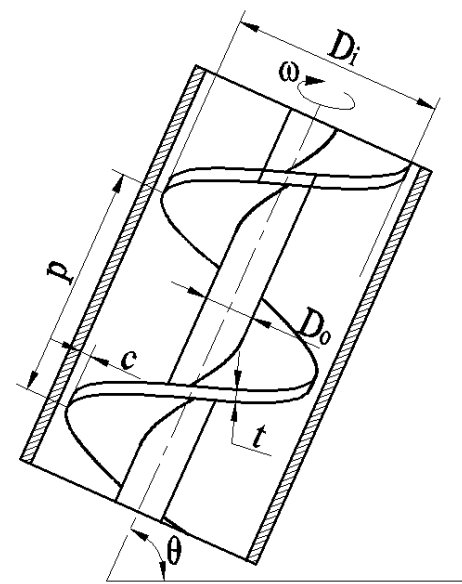

Figure 8. The size parameters of the screw conveyer

Then, the volumetric efficiencies of the granular pile were compared under different operating conditions. There are two influencing factors of the volumetric efficiency: the fill rate and the relative slippage efficiency. Thus, the volumetric efficiency of the granular pile can be expressed as:

$$
\chi=\varepsilon \psi
$$

Substituting Equations (25) and (26) into Equation (24), we have:

$Q_{T}=\frac{\pi}{4} \varepsilon \psi n\left[\left(D_{i}+2 c\right)^{2}-D_{o}^{2}\right](p-t)$

According to the relationship between mass and volume, the mass flow rate of the particles $(\mathrm{kg} / \mathrm{s})$ can be obtained as:

$Q_{m}=\frac{\pi}{240} \varepsilon \psi \rho\left[\left(D_{i}+2 c\right)^{2}-D_{0}^{2}\right](p-t)$

where $\rho$ is the particle density.

\section{CASE STUDY}

\subsection{Fill rate}

Related to rotation velocity, pitch and inclination angle, the fill rate has a direct impact on the solution of the theoretical formula for the sand mass flow rate of the screw conveyor. In the case study, the materials coming through the feed inlet are assumed to be sufficient, and the weight of the materials at the outlet were measured by an electronic scale. The test device was stopped once the reading tended to be stable. At this time, the mass of the conveyed sand is denoted as $\mathrm{m} 1$, and the maximum sand mass of the holding device, depending on the size of the device, is denoted as $\mathrm{m} 2$. In this case, the fill rate can be expressed as:

$\varepsilon=\frac{m_{1}}{m_{1}+m_{2}}$

According to the change of the fill rate with the rotation velocity in Figure 9, there is a negative linear correlation between the fill rate and the velocity. Then, the pitches were selected in light of the pitch-radius ratios: $40 \mathrm{~mm}$ for $4 / 8$, $50 \mathrm{~mm}$ for $5 / 8,60 \mathrm{~mm}$ for $6 / 8,70 \mathrm{~mm}$ for $7 / 8$ and $80 \mathrm{~mm}$ for $8 / 8$. The ratios can be expressed by Equation (30) below. The variation of the fill rate with the pitches is displayed in Figure 10 .

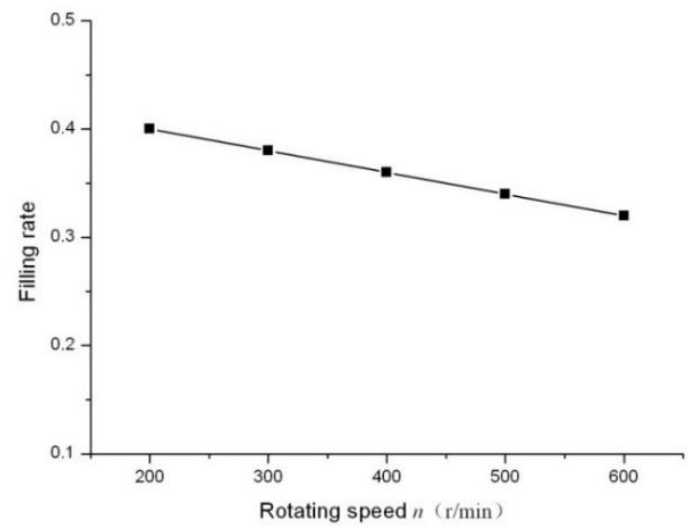

Figure 9. Variation of fill rate with velocities

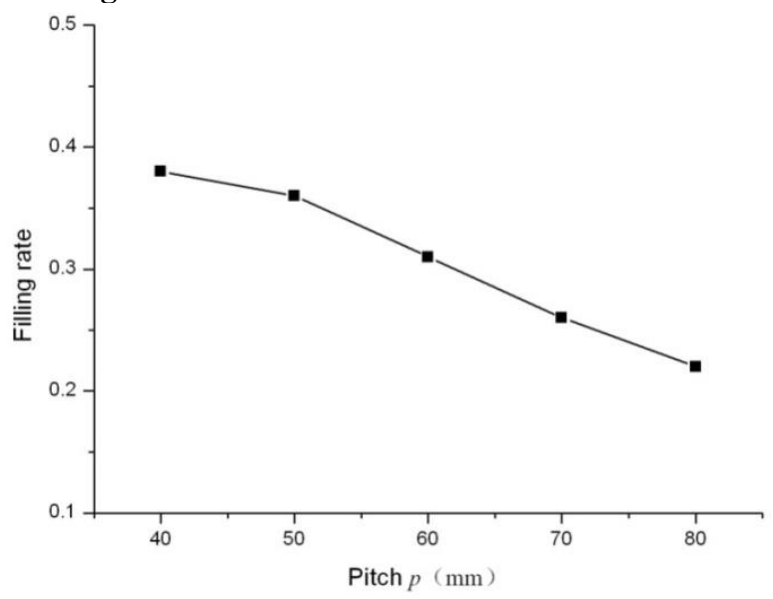

Figure 10. Variation of fill rate with pitches

$\xi=\frac{p}{D_{i}}$

The tests demonstrate a gradual decline in the fill rate with the increase of $\xi$. The decline was $5 \%$ as the $\xi$ grew from $4 / 8$ to $5 / 8,13.9 \%$ as the $\xi$ grew from $5 / 8$ to $6 / 8$, and $15.3 \%$ as the $\xi$ grew from $6 / 8$ to $7 / 8$ and $8 / 8$.

\subsection{Outlet mass flow rate vs. rotation velocity}

This section discusses the variation of outlet mass flow 
rate with rotation velocities $(200 \mathrm{r} / \mathrm{min}, 300 \mathrm{r} / \mathrm{min}, 400 \mathrm{r} / \mathrm{min}$ and $500 \mathrm{r} / \mathrm{min}$ ) at the pitch of $50 \mathrm{~mm}$ and the inclination angle of $40^{\circ}, 45^{\circ}, 50^{\circ}$ and $55^{\circ}$. Figure 11 compares the theoretical and measured outlet mass flow rates. As can be seen from the

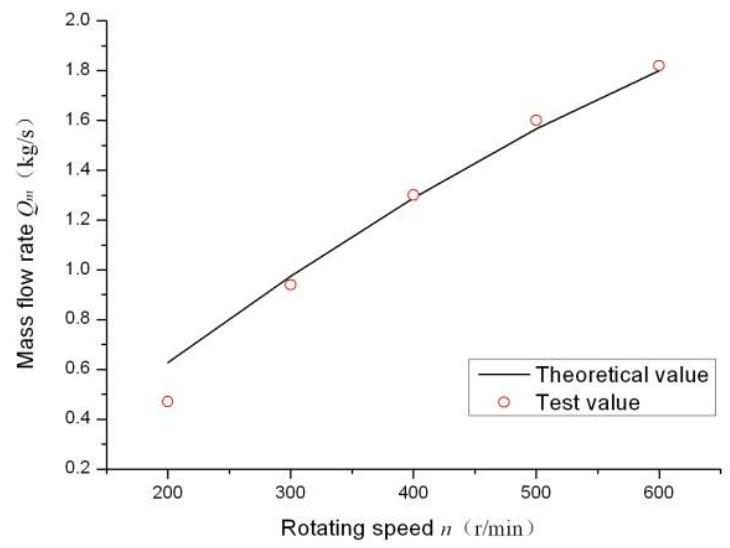

(a) 40 degrees

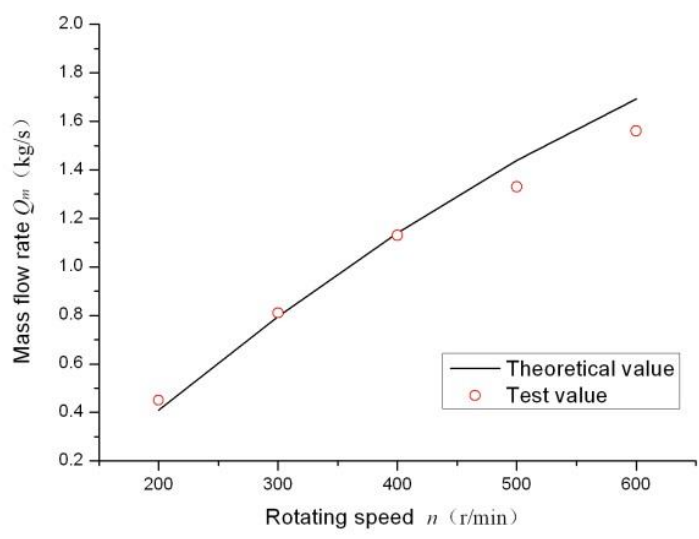

(c) 50degrees figure, the outlet mass flow rate of the screw conveyor always increased with the rotation velocity at different inclination angles, and the theoretical outlet mass flow rate agrees well with the measured result.

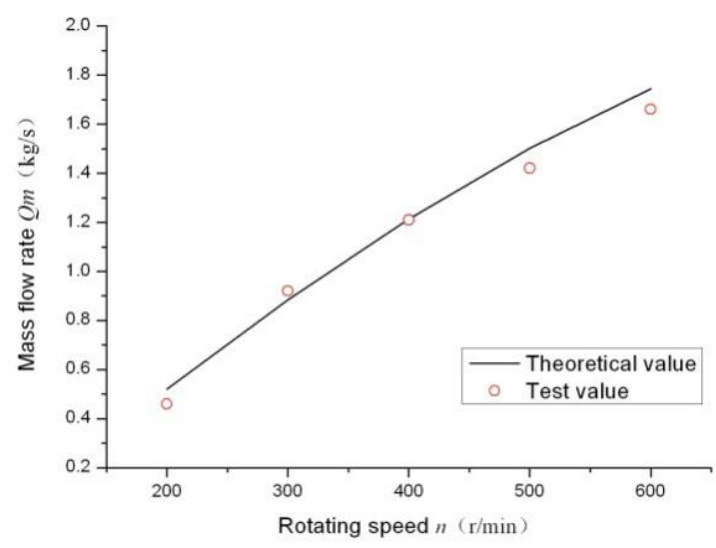

(b) 45 degrees

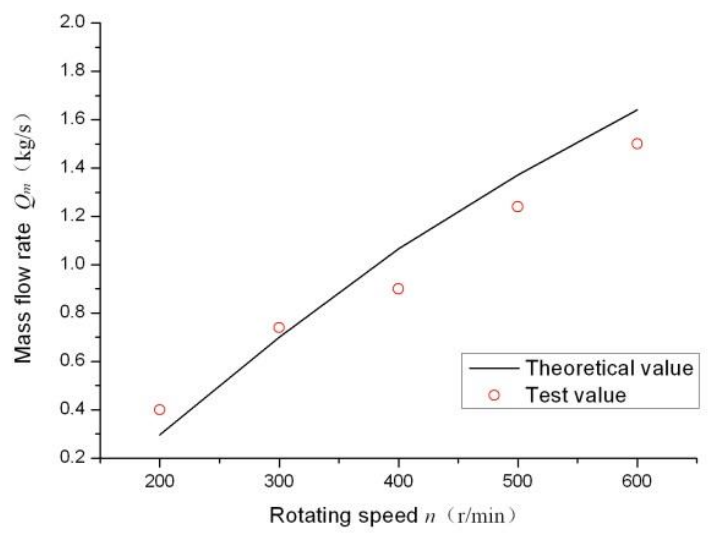

(d) 55degrees

Figure 11. Variation of outlet mass flow rate with rotation velocities at different inclination angles

\subsection{Outlet mass flow rate vs. inclination angle}

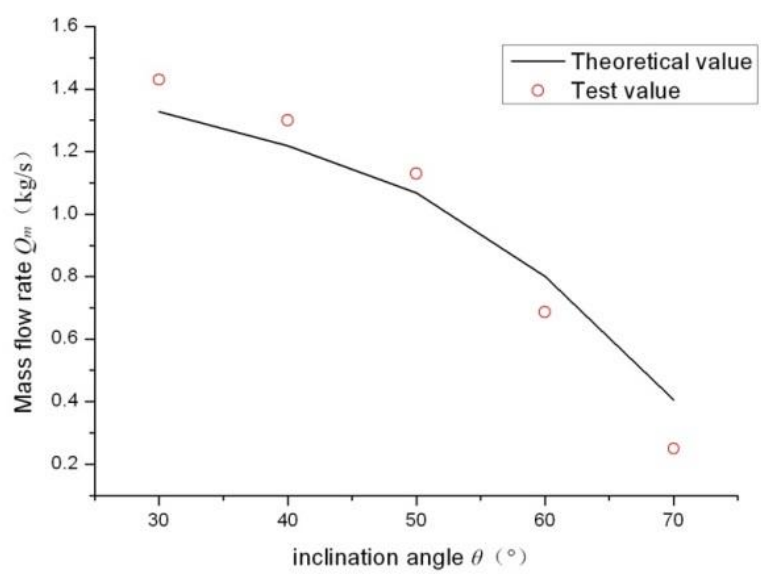

Figure 12. Variation of outlet mass flow rate with inclination angles

This section examines the variation of outlet mass flow rate with inclination angles at the pitch of $50 \mathrm{~mm}$ and the rotation velocity of $400 \mathrm{r} / \mathrm{min}$. According to the results in Figure 12, the outlet mass flow rate decreased at an increasing rate with the expansion of the inclination angle. The measured data show that: the outlet mass flow rate dropped by $0.13 \mathrm{~kg} / \mathrm{s}(9.1 \%)$ as the inclination angle expanded from $30^{\circ}$ to $40^{\circ}$; the decline of the outlet mass flow rate was $0.17 \mathrm{~kg} / \mathrm{s}(13.1 \%)$ as the inclination angle widened from $40^{\circ}$ to $50^{\circ}$; the decline was $0.443 \mathrm{~kg} / \mathrm{s}(39.2 \%)$ as the inclination angle grew from $50^{\circ}$ to $60^{\circ}$; the reduction was $0.437 \mathrm{~kg} / \mathrm{s}(63.6 \%)$ as the inclination angle increased from $60^{\circ}$ to $70^{\circ}$. The theoretical outlet mass flow rate still agrees well with the measured result.

\subsection{Outlet mass flow rate vs. pitch}

This section investigates the variation of outlet mass flow rate with pitches at the rotation velocity of $400 \mathrm{r} / \mathrm{min}$ and the inclination angles of $40^{\circ}, 45^{\circ}, 50^{\circ}$ and $55^{\circ}$. The measured results are presented in Figure 13. Figure 13(a) describes the variation of the outlet mass flow rate with pitches at the inclination angle of $40^{\circ}$. It can be seen that the outlet mass flow rate increased at a decreasing rate as the pitch-diameter ratio $\xi$ changed from $4 / 8$ to $6 / 8$; the outlet mass flow rate reached its peak at the $\xi$ of $6 / 8$; any further increase of $\xi$ led 
to the decline in the outlet mass flow rate. Similarly, Figures 13(b), 13(c) and 13(d) respectively illustrate the variation of the outlet mass flow rate with pitches at the inclination angles of $45^{\circ}, 50^{\circ}$ and $55^{\circ}$. In these cases, the outlet mass flow rate

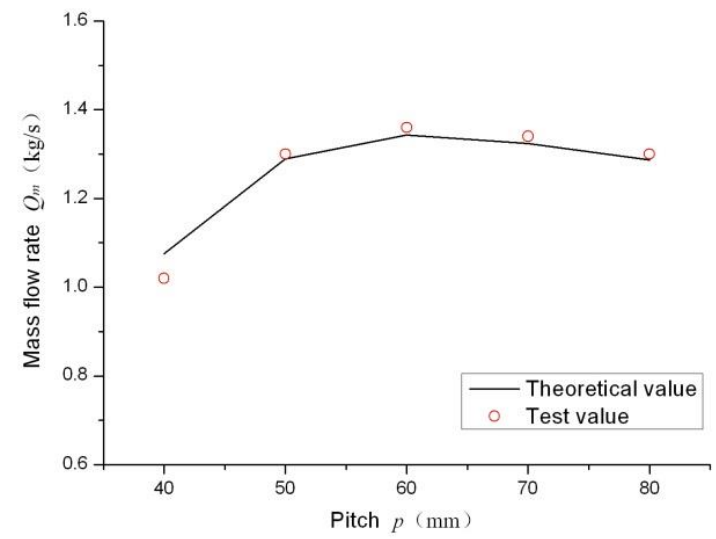

(a)40 degrees

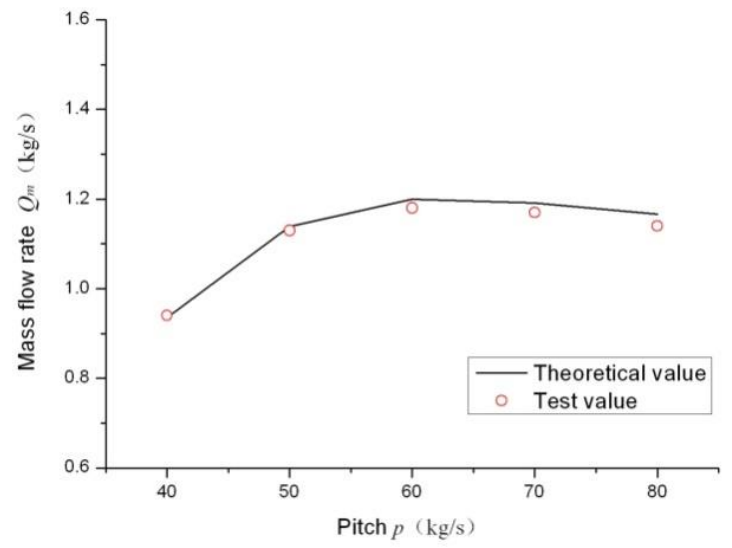

(c)50 degrees reached the peak values at the $\xi$ of $7 / 8,5 / 8$ and $6 / 8$, respectively. Thus, the maximum outlet mass flow rate can be obtained through rational setting of the pitch-diameter ratio, under the same rotation velocities.

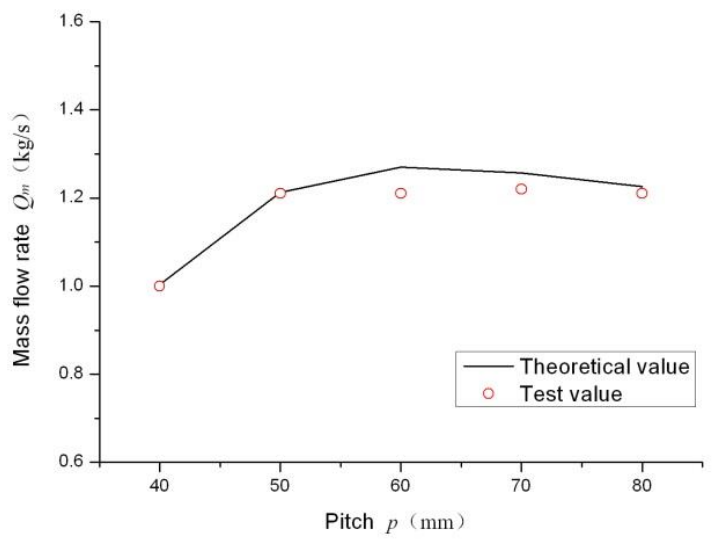

(b) 45 degrees

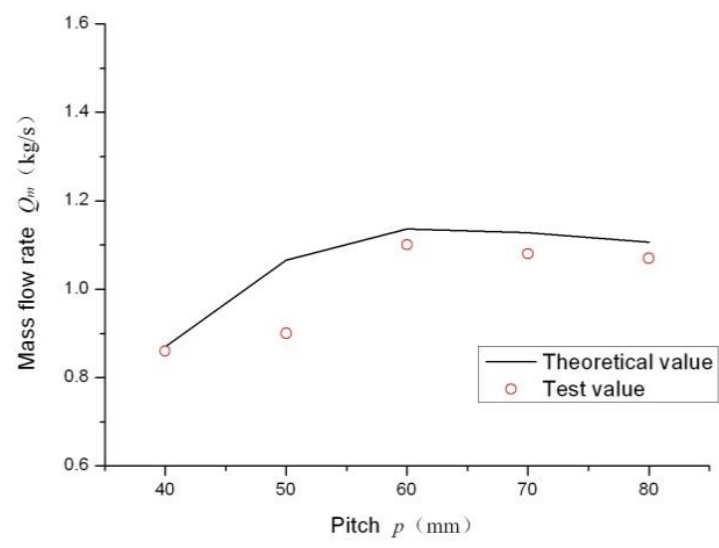

(d)55 degrees

Figure 13. Variation of outlet mass flow rate with pitches

\subsection{Application}

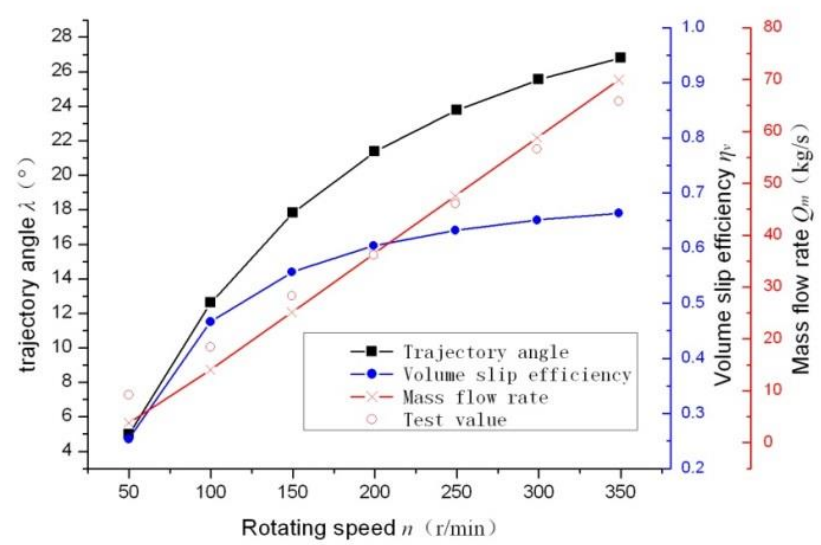

Figure 14. Comparison between the theoretical results and experimental results on the outlet mass flow rate

Taking the HS60 screw conveyor in Reference [11] as an example, the inclination angle is $45^{\circ}$, the inner diameter of the cylinder wall is $229 \mathrm{~mm}$, the diameter of the spiral blade is $218 \mathrm{~mm}$, the pitch is $175 \mathrm{~mm}$, the diameter of the screw shaft is $0.076 \mathrm{~mm}$ and the rotation velocity is between $50 \mathrm{r} / \mathrm{min}$ and $350 \mathrm{r} / \mathrm{min}$. The conveyed material is fractured sand. The theoretical model of this paper was applied to this case, and the results were contrasted with the experimental results in Reference [11]. As shown in Figure 14, With the increase of the rotating speed of the spiral axis, the trajectory angle of the particle motion increases and the increase rate gradually decreases. The volume slip efficiency of the granular reactor gradually increases and the increase rate gradually decreases with the increase of speed. Conveyer outlet mass flow rate increases with the increase of rotational speed. The theoretical model agrees well with the experiment on the variation of the outlet mass flow rate.

\section{CONCLUSIONS}

The theoretical analysis shows that the trajectory angle of particle motion is related to the inclination angle, rotation velocity and pitch of the screw conveyor. The trajectory angle varies with the radiuses of the granular pile. It is greater for the particles far from the axis of the spiral shaft than those near the axis. In summary, the trajectory angle is positively correlated with the rotation velocity and pitch and 
negatively with the inclination angle.

The fill rate has a linear negative correlation with the rotation velocity and a negative correlation with the pitchdiameter ratio.

The outlet mass flow rate decreases with the increase of the inclination angle and increases with the rotation velocity. However, once the rotation velocity reaches a certain level, any further increase will not enhance the flow rate.

Under different inclination angles, the pitch-diameter ratio has a major impact on the outlet mass flow rate. Besides, there exists an optimal pitch-diameter ratio that maximizes the outlet mass flow rate.

The change law obtained by our theoretical model was contrasted with that observed in Reference [3]. The comparison shows that the two laws are basically the same. Thus, the proposed model is accurate enough to be applied in the practical design of the screw conveyor.

\section{ACKNOWLEDGMENT}

The authors would like to thank all members for their support. The authors also would like to acknowledge all members of the project team. Financial support for this study, provided by National Key R \& D project (2016YFC0303703) are gratefully acknowledged.

\section{REFERENCES}

[1] Deng BQ, Chen H. (2011). Study on the development trend of the mixed sand truck. China Petroleum and Chemical Standards and Quality 07: 193. https://doi:10.3969/j.issn.1673-4076.2011.07.161

[2] Liu C. (2013). Model test similarity design and numerical simulation of the spiral sand conveyor. China Petroleum Machinery 1:55-58. https://doi: 10.3969/j.issn.1001-4578.2013.01.014

[3] Weiling A, Jin GL, Zhu ZC. (1964). Research on the conveying process of vertical screw conveyor. Lifting transport machinery 12(2): 18-22.

[4] Song R. (1993). Conveying mechanism of horizontal screw conveyor. Journal of Wuhan University of Technology [Transportation Science \& Engineering] 03.

[5] Huang SM. (1998). Screw conveyor transport mechanism and the determination of its main parameters. Paper Science \& Technology (3): 27-31.

[6] $\mathrm{Hu}$ YK, Dai LL. (2000). Theories and calculation of the auger -type conveyer. Journal of Nanchang University (Engineering 22(4): Edition) 29-33. https://doi:10.3969/j.issn.1006-0456.2000.04.008

[7] XuYW. (2008). Selection of design parameters of screw conveyor. Brick World (7): 32-37.

[8] Xiang DZ, Xu YW. (2010). Design parameter selection of spiral conveyer. Cement Technology. https://doi: 10.3969/j.issn.1001-6171.2010.01.004

[9] $\mathrm{Xu} \mathrm{Y.} \mathrm{(2008).} \mathrm{The} \mathrm{choice} \mathrm{and} \mathrm{decision} \mathrm{of} \mathrm{design}$ parameters for screw conveyor for rubber industry. China Rubber/plastics Technology \& Equipment.

[10] Nilsson PJI. (1971). On the vertical screw conveyor for non-cohesive bulk materials. Acta Polytechnical Scadinavica Mech. Eng. Sevies 64: 1-96.

[11] li 1, hu y, wu h. (2010). Analysis and optimization of sand transporting capacity of spiraling sand blender.

Petroleum Machinery 38(6): 96-98. https://10.16082/j.cnki.issn.1001-4578.2010.06.001

[12] Jin R, Yan M. (1995). Design of large-dip screw conveyor and parameter selection. Inner Mongolia Highway and Transport, 43: 37-39.

[13] Roberts AW. (1999). The influence of granular vortex motion on the volumetric performance of enclosed screw conveyors. Powder Technology 104(1): 56-67. https://doi.org/10.1016/S0032-5910(99)00039-XGet rights and content

\section{NOMENCLATURE}

$V_{B}$

$V_{R}$

$V_{A}$

$r$

$\omega$

$n$

$V_{Z}$

$V_{F}$

$p$

$\rho$

$h$

$p_{r}$

$k_{j}$

$p_{v}$

$F_{a}$

$k_{s}$

W

$R_{c}$

$m$

$F_{c}$

$F_{N}$

$F_{a}$

$F_{D}$

$\mu_{g}$

$\theta$

$\Phi s$

$r_{i}$

$\lambda_{i}$

$\lambda_{a v}$

$\chi$

$V_{L z}$

$Q$

$Q_{T}$

$D_{i}$

$D_{o}$ $p$

$t$

c circumferential velocity of the spiral blade velocity of particles relative to the blade surface

absolute velocity of the particles distance from the centre of the screw shaft angular velocity of the screw shaft rotation velocity of the screw shaft ( $\mathrm{r} / \mathrm{min}$ ) effective delivery velocity along the axis of the spiral shaft

rotation velocity along the tangent line

vertical pressure

density of particles

height of the granular pile from the free surface

lateral positive pressure of the granular pile lateral pressure coefficient of the granular pile

vertical pressure

lateral positive pressure of the ring at low rotation velocities

Stress side pressure coefficient

gravity of a circular ring unit of the granular pile

maximum radius of the ellipse

mass of the sand in a ring segment

centrifugal force of the ring segment during the circular motion

pressure of the granular pile against the cylinder wall during the steady flow of the particles

static lateral pressure

boundary friction force

Friction of coefficient boundary

inclination angle

friction angle of Particle

distance of the circular ring unit from the spiral shaft

trajectory angle of the unit at the distance of $r_{i}$.

final trajectory angle of the particles

efficiency of the relative slippage

maximum theoretical conveying velocity volumetric flow rate of the screw conveyor theoretical maximum mass flow rate of the screw conveyor

diameter of the blade edge

diameter of the spiral shaft

pitch

blade thickness

blade-wall clearance 
